\section{An unusual case of familial hypertrophic cardiomyopathy with left ventricular systolic dysfunction: a still unsolved diagnosis}

\author{
Elena Biagini, ${ }^{1}$ Chiara Pazzi, ${ }^{1}$ \\ Stefania Rosmini, ${ }^{1}$ Ornella Leone, ${ }^{2}$ \\ Domenico A. Coviello, ${ }^{3}$ Claudio Rapezzi ${ }^{1}$ \\ ${ }^{1}$ Institute of Cardiology and ${ }^{2}$ Department \\ of Pathology, S. Orsola-Malpighi \\ University Hospital, Bologna; ${ }^{3}$ Human \\ Genetic Laboratory, Galliera Hospital, \\ Genoa, Italy
}

\section{Abstract}

A 35-year-old woman was referred to our centre for clinical management of hypertrophic cardiomyopathy (HCM) with left ventricular (LV) systolic dysfunction (end-stage evolution). She was recently diagnosed elsewhere because of palpitations. Her 7-year-old daughter underwent familiar screening and she was diagnosed with classic HCM. She was completely asymptomatic without extracardiac or systemic manifestations. During the following years, they both experienced a similar clinical course with worsening dyspnoea and progressive deterioration of LV systolic function. They both underwent heart transplantation, the mother at the age of 47 and the daughter at the age of 23, respectively. Many diagnostic hypotheses, including sarcomeric HCM, Anderson-Fabry disease, glycogen storage diseases and mitochondrial cardiomyopathies have been taken into account. The diagnostic work-up included serial electrocardiogram and echocardiographic assessments, pathologic evaluation of the explanted hearts and genetic analysis of 8 sarcomeric and 3 metabolic genes. Even if a shared HCM phenotype (LV systolic dysfunction in two first-degree female family members associated with a likely autosomal dominant inheritance and absence of extracardiac or multisystemic manifestations) could support a temptative diagnosis of sarcomeric HCM, a definitive diagnosis could not be reached, due to the lack of genetic analysis to confirm such diagnosis.

\section{Case Report}

A 35-year-old woman was referred to our centre for clinical management of hypertrophic cardiomyopathy (HCM) with left ventricular (LV) systolic dysfunction (end-stage phase). This condition was recently diagnosed in a different hospital because of palpitations.

The electrocardiogram (ECG) showed sinus rhythm and negative $\mathrm{T}$ waves in the lateral leads (Figure 1A). Echocardiography showed LV hypertrophy (maximal wall thickness 14 $\mathrm{mm}$ ) associated with LV enlargement (enddiastolic diameter $56 \mathrm{~mm}$ ) and $\mathrm{LV}$ ejection fraction (EF) of $30 \%$. Of note, a relative wall thinning localized at the basal segment of the interventricular septum with marked akinesia was present (Figure 1B and 1C, arrows). At that time she was mildly symptomatic, showing dyspnoea on mild efforts (NYHA functional class II). No other clinical manifestations were present and laboratory parameters were unremarkable. Medical therapy with beta-blockers and ACE-inhibitors was started.

The patient's 7-year-old daughter underwent familiar screening with ECG and echocardiography. She was completely asymptomatic. No mental retardation or other extracardiac manifestations were detected and laboratory parameters were normal. The ECG showed extreme LV hypertrophy with infero-lateral pseudonecrosis and negative $\mathrm{T}$ waves in the infero-lateral leads (Figure 2A). Two-dimensional echocardiography disclosed a classical form of HCM with a maximal wall thickness of $16 \mathrm{~mm}$ at the postero-lateral wall level, a $\mathrm{LV}$ end-diastolic diameter of $35 \mathrm{~mm}$ and a LV ejection fraction of $78 \%$. As the mother, a relative and localized wall thinning associated with hypo-akinesia at the basal interventricular septum level was present (Figure 2C, arrow).

Question: On the basis of these clinical and instrumental findings, what is the most likely diagnosis? Is there any clue or clinical marker suggesting specific hypotheses?

Reply: Expert cardiologist (Prof. Claudio Rapezzi)

Clinical hypotheses: i) Sarcomeric HCM; ii) Glycogen storage diseases; iii) Anderson-Fabry disease; iv) Mitochondrial diseases. All these diagnostic hypotheses should be taken into account in the assessment of idiopathic LV hypertrophy. ${ }^{1}$ Some clues can help to reach the final diagnosis:-2-5 i) Both the mother and the daughter show two different HCM phenotypes, the first associated with LV systolic dysfunction and the second with a classic form of HCM. Moreover they both share a particular echocardiographic aspect of hypo-akinesia of the basal interventricular septum (with relative reduced thickness), supporting the hypothesis of a common disease. Therefore, they are both likely affected by the same disease; ii) The transmission of the disease could be autosomal dominant. The presence of two female family members affected in two consecutive generations makes an autosomal recessive form unlikely. Additionally, a female to
Correspondence: Elena Biagini, Institute of Cardiology, S. Orsola-Malpighi Hospital, University of Bologna, via Massarenti n. 9, 40125 Bologna, Italy.

Tel: +39.051 .6364483 - Fax: +39.051 .344859 .

E-mail: biaginielena@libero.it

Key words: education, familial hypertrophic cardiomyopathy phenotype, left ventricular systolic dysfunction, extensive myocardial fibrosis, cardiogenetics.

Received for publication: 17 May 2012.

Revision received: 2 July 2012.

Accepted for publication: 4 July 2012.

This work is licensed under a Creative Commons Attribution NonCommercial 3.0 License (CC BYNC 3.0).

CC Copyright E. Biagini et al., 2012

Licensee PAGEPress, Italy

Cardiogenetics 2012; 2:e8

doi:10.4081/cardiogenetics.2012.e8

female transmission almost excludes an Xlinked inheritance. A matrilinear inheritance (i.e. mitochondrial inheritance) is possible, but it is unlikely due to the lack of extracardiac manifestations in both patients. Therefore, Andreson-Fabry disease (X-linked), Danon disease (X-linked) or mitochondrial cardiomyopathies (maternal inheritance) are unlikely to represent the underlying diagnosis; iii) No extracardiac or mutlisystemic manifestations are present, such as renal insufficiency, acroparesthesias and angiokeratomas (typical of Fabry disease $)^{3}$ or neurological, musculoskeletal or ocular involvement (suggestive of a mitochondrial disease); ${ }^{5}$ iv) Finally any pre-excitation (typical of glycogen storage diseases or mitochondrial diseases) are evident in both ECGs. ${ }^{4,5}$ Considering the phenotype similarity between the mother and the daughter, the potential autosomal dominant transmission and the lack of typical involvement of other organs, the most likely diagnosis is sarcomeric HCM.

\section{Case Report: follow up}

During the following years, the mother experienced a progressive increase of dyspnoea associated with marked LV dysfunction (ejection fraction 26\%) and mitral and tricuspid regurgitations with pulmonary hypertension at echocardiography. The rapid end stage disease progression required heart transplantation when she was 47 years old.

Despite optimal medical treatment with betablockers and ACE-Inhibitors, the daughter had a similar clinical course. At 23 years of age 
ECG showed reduced voltages and QRS enlargement compared to the previous one, infero-lateral necrosis and antero-lateral ST segment elevation with biphasic $\mathrm{T}$ waves in the lateral leads were also present (Figure 2B). Echocardiography showed severe LV enlargement with preserved areas of LV hypertrophy (maximum $\mathrm{LV}$ wall thickness of $16 \mathrm{~mm}$ ) and severe reduction of $\mathrm{LV}$ systolic function with ejection fraction of $32 \%$ (Figure 2D). Moreover moderate mitral regurgitation, mild tricuspid regurgitation and pulmonary hypertension were detected. Cardiac magnetic resonance imaging confirmed LV hypertrophy associated with severe $\mathrm{LV}$ dilatation (LV end-diastolic volume $196 \mathrm{~mL} / \mathrm{m}^{2}$ and EF 28\%) and showed widespread subepicardial and transmural areas of late gadolinium enhancement suggesting intramyocardial fibrosis. At ECG Holter monitoring frequent episodes of non-sustained ventricular tachycardia were found. Two months later, due to development of progressive dyspnoea and clinical deterioration she underwent heart transplantation.

Pathologic assessment of the explanted hearts evidenced a similar heart weight (510 vs 490 g). However the mother's heart was more rounded and enlarged (Figure 3A) compared to the daughter's heart (Figure 3B). Short axis slices at mid-ventricular level of mother's heart showed LV enlargement, hypertrophy of the septum, mild hypertrophy of the antero-lateral wall and thinned areas at the posterior wall (Figure 3C). Short axis slices at mid-ventricu- lar level of daughter's heart showed marked LV hypertrophy of the septum, mild hypertrophied areas at antero-lateral wall and a relative thinning of inferior wall (Figure 3D).

Extensive fibrosis throughout the septum and the left ventricle was present in both hearts. In the mother's heart (Figure 3C), fibrosis was localized at the anterior and inferior left ventricular walls. In the daughter's heart (Figure 3D) fibrosis was more extensive and circumferentially distributed throughout the left ventricle.

Histology showed similar findings in both hearts (Figure 4): myocyte hypertrophy and multiple myocardial disarray (Figures 4A-4B), extensive and marked sarcoplasmic vacuolization of the myocytes (Figures 4C), extensive
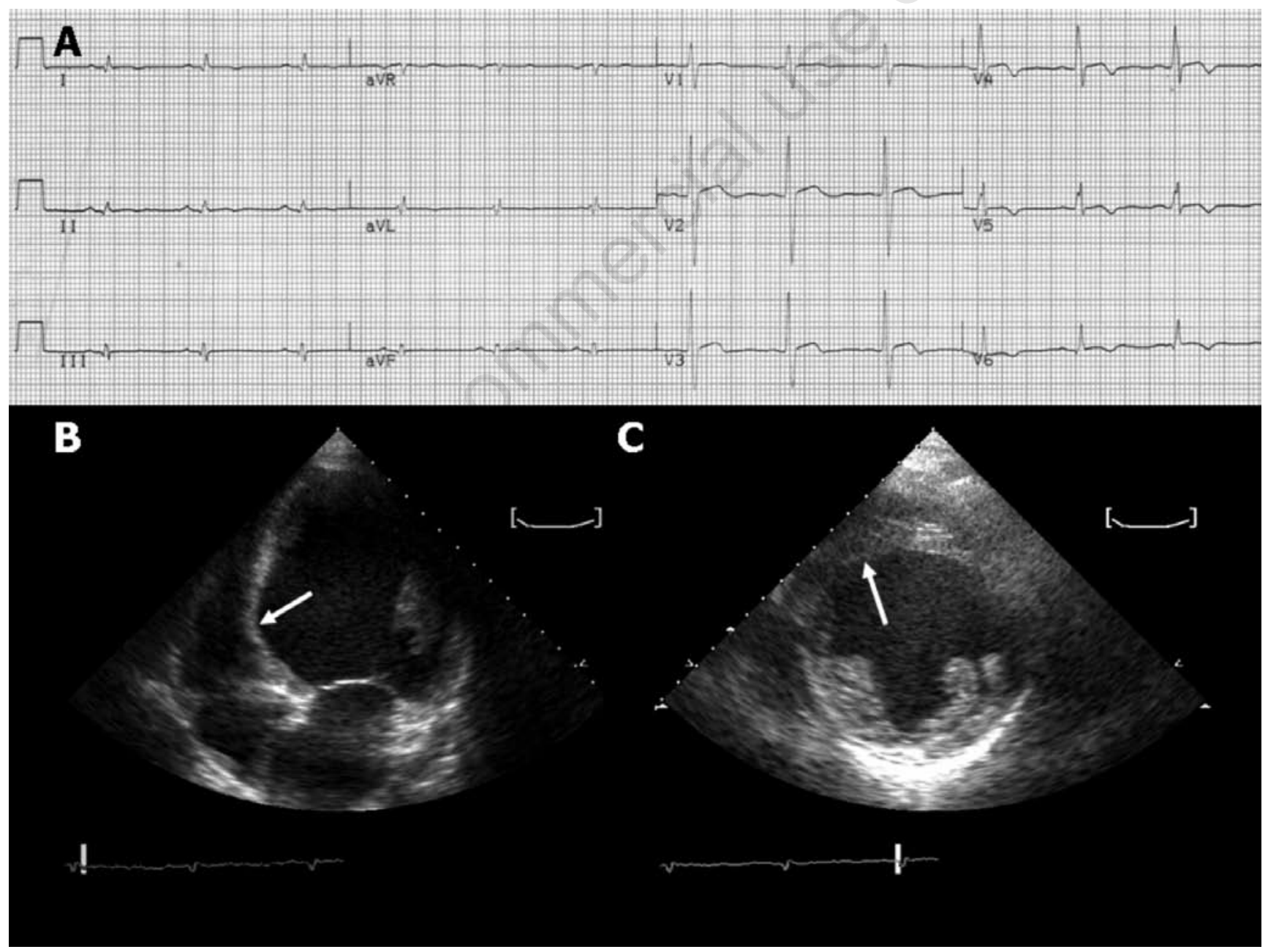

Figure 1. Electrocardiogram (ECG) and echocardiogram of the mother. A) ECG showing sinus rhythm and negative T waves in the lateral leads. B) Apical 4-chambers view showing left ventricular (LV) hypertrophy and LV enlargement. C) Parasternal short axis view at level of papillary muscles showing LV hypertrophy and enlargement. Of note, a relative and localized wall thinning at the basal segment of the interventricular septum was present (white arrow). 
replacement fibrosis (Figures 4D-4E) and mild to moderate vasculopathy of small mural vessels (Figure 4F) (only mild and focal parietal thickening due to intimal hyperplasia or medial hypertrophy) were present.

Question: On the basis of these new information, specifically the observation that both patients have hypertrophic cardiomyopathy associated with left ventricular systolic dysfunction, and on the basis of the macroscopic and histological examination of the explanted hearts, what is the most likely diagnosis?

Reply: Expert pathologist (Dr. Ornella Leone)

On the basis of the macroscopic and histologic pathological examination, the most likely diagnosis is sarcomeric end-stage HCM, even though both cases show some atypical histological aspects: i) unusually marked and widespread myocyte vacuolization and ii) mild focal changes in small mural vessels (usually prominent in end-stage HCM).

Even if hypertrophic vacuolated myocytes are commonly seen in end-stage HCM, such widespread vacuolization could suggest a diagnosis of glycogen storage disease. Nevertheless Pas and Pas-d stainings on paraffin sections showing no glycogen storage (although frozen sections are preferable for these stainings, as glycogen may wash out with routine processing) make this hypothesis unlikely. Moreover ultrastructural examination of the daughter's heart showed no intracellular accumulation products and nor electron dense concentric lamellar bodies (typical of, but not exclusive to, Anderson-Fabry disease). All these findings make the alternative diagnosis of storage cardiomyopathies (CMPs) definitely improbable. At ultrastructural assessment, widespread mitochondrial proliferation/giant mitochondria/mitochondrial inclusions typical of mitochondrial CMPs were also absent. Moreover mitochondrial CMPs usually show a granular cytoplasmic appearance together with lack of myocardial disarray. No one of these findings was present. Finally, the macroscopic and histological images were not consistent with amyloid infiltration, and this was easily excluded with red congo stain.
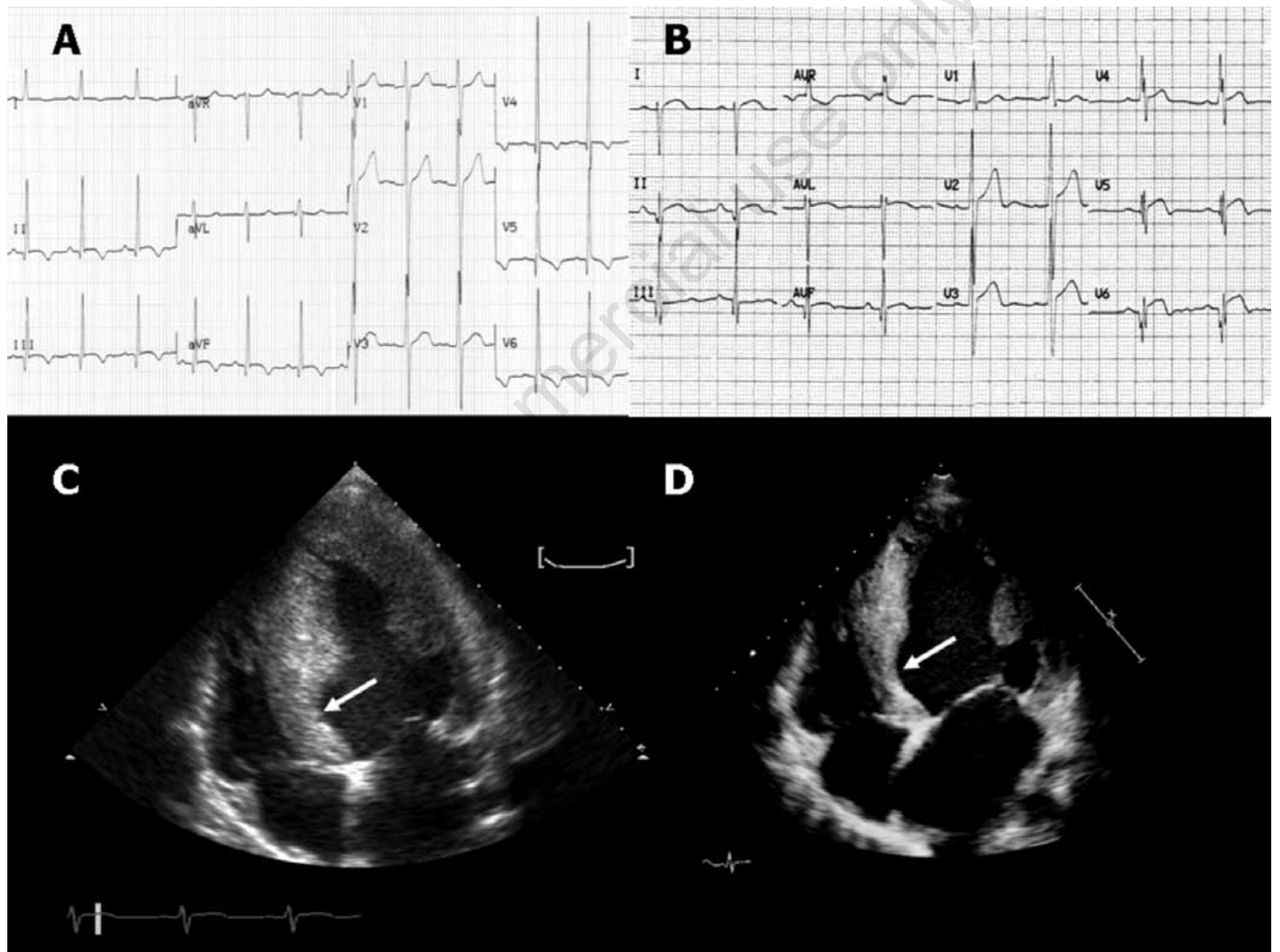

Figure 2. Electrocardiograms (ECG) and echocardiograms of the daughter. A) ECG at the age of 7 years old (halved voltages). Extreme left ventricular (LV) hypertrophy and infero-postero-lateral necrosis with negative $T$ waves in the infero-lateral leads. B) ECG at the age of 23 years old (note that voltages are not halved on this occasion). Compared to the previous one, decreased voltages and QRS enlargement are evident. Infero-lateral pseudonecrosis and antero-lateral ST segment elevation with biphasic $T$ waves are present. C) Echocardiography at the age of 7 years old. The apical 4-chamber view shows marked left ventricular hypertrophy prevalent at posterolateral level with normal ventricular cavity. As the mother, a relative wall thinning localized to the basal interventricular septum is evident (white arrow). D) Echocardiohrapy at the age of 23 years old. Compared to the previous one, the apical 4-chamber view shows thinned LV walls and LV enlargement. The aspect of thinness of the basal interventricular septum is still evident (white arrow). 


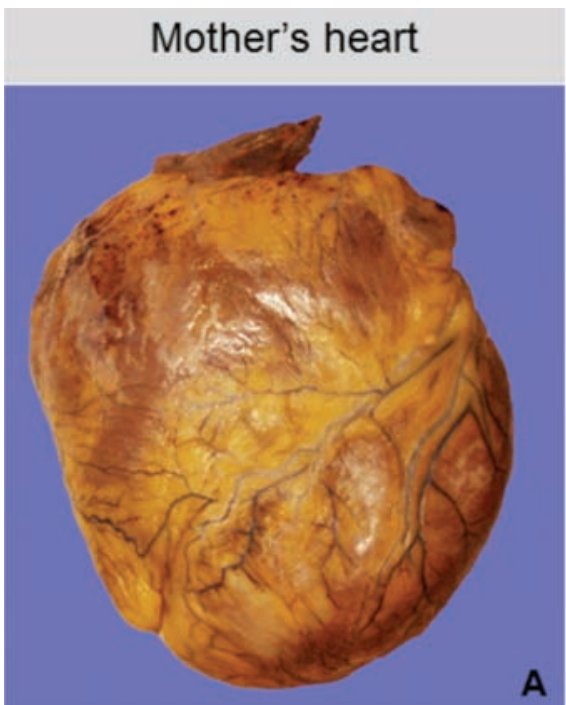

\section{Daughter's heart}

A
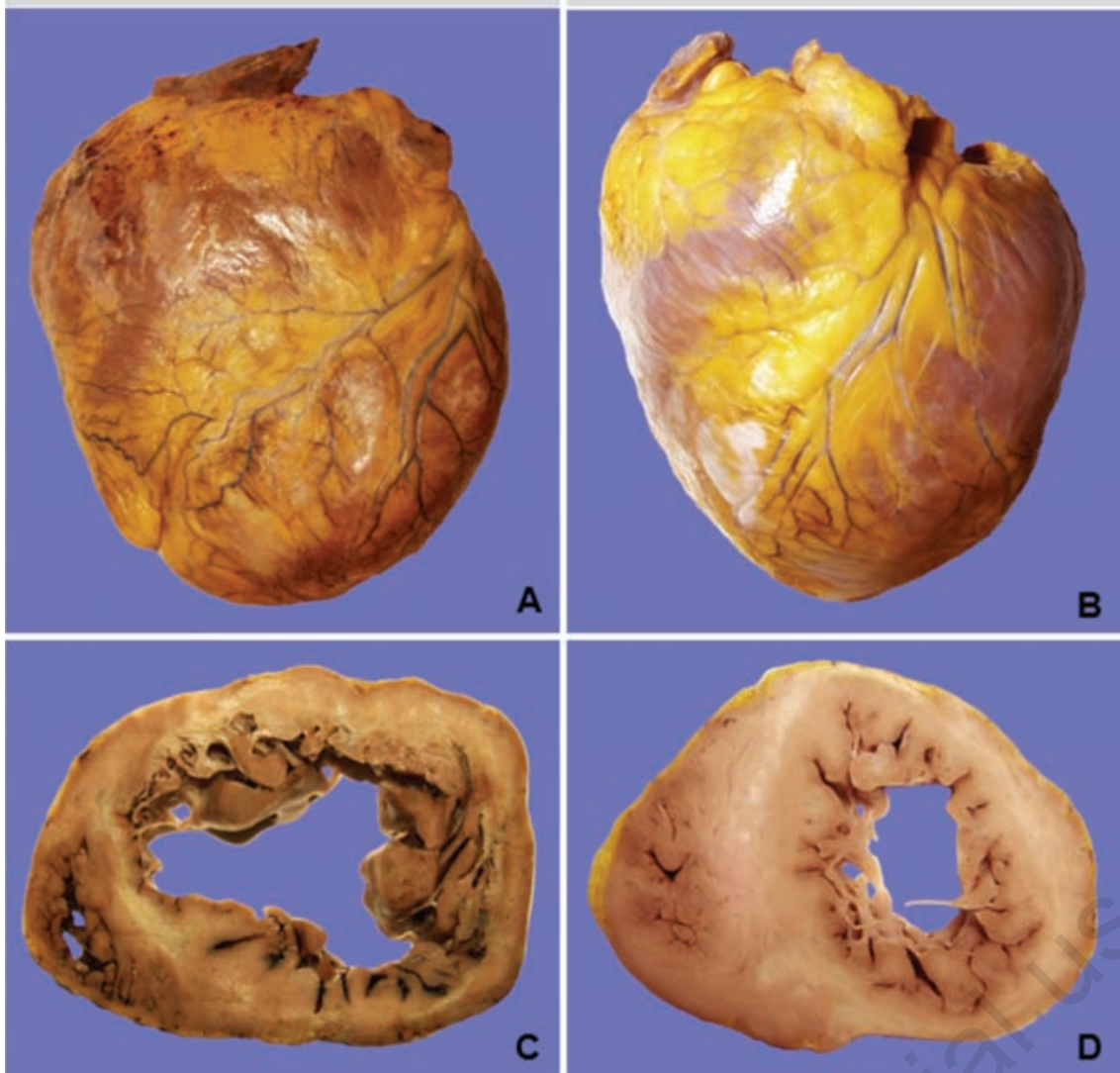

Figure 3. Pathologic assessment of the explanted hearts. A) Mother's explanted heart. Macroscopic anterior view showing an enlarged and rounded heart. B) Daughter's explanted heart. Macroscopic anterior view showing a less enlarged and less rounded heart, compared to the mother's one. C) Short axis slice of the mother's heart at mid-ventricular level showing left ventricular (LV) dilatation and a LV hypertrophy pattern characterized by hypertrophy of the septum, mildly hypertrophied areas in the antero-lateral wall and thinned areas in the posterior wall. The anterior right ventricular wall is also hypertrophic. D) Short axis slice of the daughter's heart at mid-ventricular level showing marked LV hypertrophy (maximum wall thickness of $25 \mathrm{~mm}$ at the level of the septum), mildly hypertrophied areas in the antero-lateral wall and thinned inferior wall. Diffuse hypertrophy of the right ventricular myocardium is also evident.
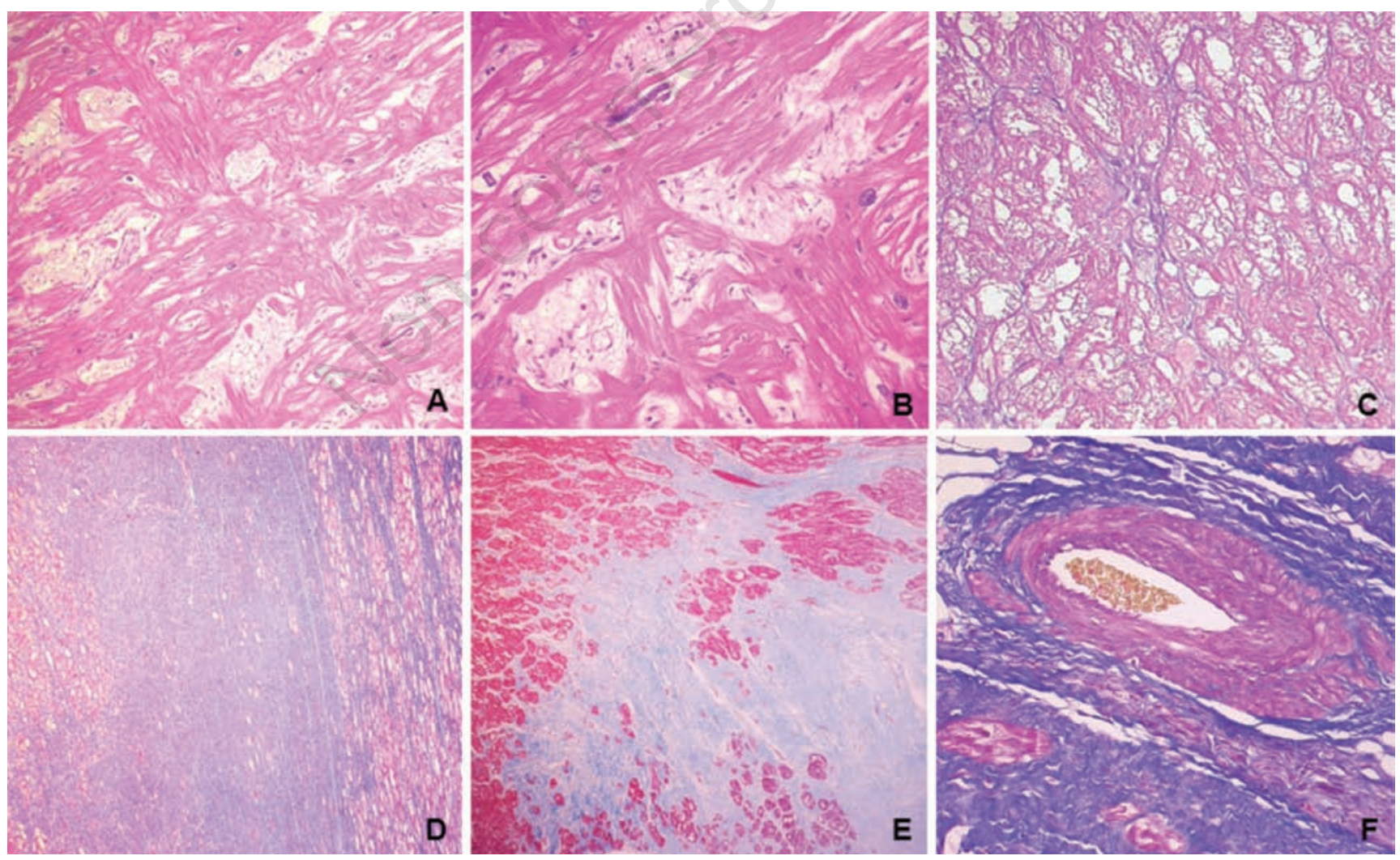

Figure 4. Hystologic assessment of both hearts showing myocyte hypertrophy and multiple myocardial disarray (A-B), extensive and marked sarcoplasmic vacuolization of the myocytes $(\mathrm{C})$, extensive replacement fibrosis (D-E) and mild to moderate vasculopathy of small mural vessels $(F)$. 


\section{Case Report: end}

In both patients 8 sarcomeric and 3 metabolic genes were tested. In particular MYH7 (cardiac myosin heavy chain beta), MYBPC3 (cardiac myosin binding protein $\mathrm{C}$ ), TNNT2 (cardiac Troponin T), TNNI3 (cardiac Troponin I), TPM1 (tropomyosin alfa - 1), ACTC1 (cardiac actin alpha), MYL3 (myosin essential light chain) and MYL2 (myosin regulatory light chain) as sarcomeric genes; GLA (alpha-galactosidase A), LAMP2 (lysosome associated membrane glycoprotein 2) and PRKAG2 (AMPactivated protein kinase) as metabolic genes. A new mutation in MYL3 gene (p.Thr106Ile) and a new mutation in LAMP2 gene (p. Gly80Val) were found in the mother. Neither of these mutations are described in literature, so their pathogenic role is not proven. The analysis of the same genes in the daughter did not show any mutations. The genetic analysis was also performed on the explanted hearts and confirmed these results.

\section{Expert geneticist (Dr. Domenico A. Coviello)}

Unexpectedly no mutations were detected in the daughter. This result was confirmed by further investigations, including repetition of genetic analysis on both blood sample and on samples from both explanted hearts. A paternity test was also performed. Therefore, even if the mother and the daughter showed a very similar cardiac phenotype and clinical course, they didn't share any common mutations. The mother had two different mutations, which might be responsible for two different diseases: the mutation in MYL3 gene could be related to a sarcomeric HCM with an autosomal dominant inheritance, while the mutation in the LAMP2 gene may correlate with a lysosomal glycogen storage disease (Danon disease) with an X-linked inheritance. However, none of these mutations have been previously described, so their pathogenetic role is not certain. Genetic analysis usually is very useful in establishing the exact diagnosis, especially in the context of HCM phenotype. However in this case the molecular analysis of the most frequently involved genes not confirm any etiological diagnosis.

\section{Conclusions}

The evidence of a shared HCM phenotype with progression to LV systolic dysfunction in two first-degree female family members associated with a likely autosomal dominant inheritance and the absence of extracardiac or multisystemic manifestations could support a diagnosis of sarcomeric HCM with end-stage evolution. However, genetic analysis failed to confirm this diagnosis.

In both patients we analyzed 8 sarcomeric genes and 3 metabolic genes. Previous studies analyzing a similar number of sarcomeric genes in large series of unrelated patients with the classic form of HCM found a positive genotype in no more than $50-60 \%$ of cases. ${ }^{6-8}$ Similar results have been reported by a recent study on patients with end-stage HCM. In particular, genetic analysis of 10 HCM-related genes disclosed a positive genotype in $58 \%$ of the patients with a $13 \%$ of double mutations. ${ }^{9}$ These results suggest that not all the genes implicated in the pathogenesis of HCM are known, yet. Therefore genetic analysis can confirm the diagnosis of HCM, but we cannot rely on it alone to exclude this disease. Genetic screening remains an essential tool in clinical practice, particularly with the aim to confirm a clinical diagnosis of HCM and perform familiar studies. ${ }^{10}$ Functional studies are needed to verify the pathogenetic role of new mutations in cases were the segregation of the mutation and the clinical phenotype within the family is not present. In addition the routine molecular diagnostic evaluation do not include all intron regions of the genes analyzed nor the 5' and the 3' UTR regions, were regulatory sequences could be mutated. The benefits of Next Generation Sequencing in terms of a more comprehensive genetic analysis (more extended set of genes, including genetic modifiers) with a potential time-costs optimization, will be potentially striking for HCM and inherited heart diseases, in general. ${ }^{11}$

\section{References}

1. Elliott P, Andersson B, Arbustini E, et al. Classification of the cardiomyopathies: a position statement from the European Society Of Cardiology Working Group on Myocardial and Pericardial Diseases. Eur Heart J 2008;29:270-6.

2. Biagini E, Coccolo F, Ferlito $\mathrm{M}$, et al. Dilated-hypokinetic evolution of hypertrophic cardiomyopathy: prevalence, incidence, risk factors, and prognostic implications in pediatric and adult patients. $\mathrm{J}$ Am Coll Cardiol 2005;46:1543-50.

3. Elliott P, Baker R, Pasquale F, et al. Prevalence of Anderson-Fabry disease in patients with hypertrophic cardiomyopathy: the European Anderson-Fabry Disease survey. Heart 2011;97:1957-60.

4. Maron BJ, Roberts WC, Arad M, et al. Clinical outcome and phenotypic expression in LAMP2 cardiomyopathy. JAMA 2009;301:1253-9.

5. Berardo A, Musumeci 0, Toscano A. Cardiological manifestations of mitochondrial respiratory chain disorders. Acta Myol 2011;30:9-15.

6. Richard P, Charron P, Carrier L, et al. Hypertrophic cardiomyopathy: distribution of disease genes, spectrum of mutations, and implications for a molecular diagnosis strategy. Circulation 2003;107:2227-32.

7. Olivotto I, Girolami F, Ackerman MJ, et al. Myofilament protein gene mutation screening and outcome of patients with hypertrophic cardiomyopathy. Mayo Clin Proc 2008;83:630-8.

8. Van Driest SL, Ommen SR, Tajik AJ, et al. Sarcomeric genotyping in hypertrophic cardiomyopathy. Mayo Clin Proc 2005;80: 463-9.

9. Garcia-Pavia P, Vázquez ME, Segovia J, et al. Genetic basis of end-stage hypertrophic cardiomyopathy. Eur J Heart Fail 2011; 13:1193-201.

10. Monserrat L, Mazzanti A, Ortiz-Genga M, et al. The interpretation of genetic tests in inherited cardiovascular diseases. Cardio genetics 2011;1:e8.

11. Meder B, Haas J, Keller A, et al. Targeted next-generation sequencing for the molecular genetic diagnostics of cardiomyopathies. Circ Cardiovasc Genet 2011;4: $110-22$. 